\title{
Bilateral venous sinuses of Kelch
}

\author{
Mitchell Couldwell ${ }^{1}$, Aimee Aysenne ${ }^{1}$, Joe Iwanaga ${ }^{1,2}$, Aaron S. Dumont ${ }^{1}$, R. Shane Tubbs ${ }^{1,2,3,4,5,6,7}$ \\ ${ }^{1}$ Department of Neurosurgery, Tulane Center for Clinical Neurosciences, Tulane University School of Medicine, New Orleans, LA, ${ }^{2}$ Department of \\ Neurology, Tulane Center for Clinical Neurosciences, Tulane University School of Medicine, New Orleans, LA, USA, ${ }^{3}$ Department of Anatomical \\ Sciences, St. George's University, St. George's, Grenada, ${ }^{4}$ Department of Structural \& Cellular Biology, Tulane University School of Medicine, New \\ Orleans, LA, ${ }^{5}$ Department of Neurosurgery and Ochsner Neuroscience Institute, Ochsner Health System, New Orleans, LA, ${ }^{6}$ Department of Surgery, \\ Tulane University School of Medicine, New Orleans, LA, USA, ${ }^{7}$ University of Queensland, Brisbane, Australia
}

\begin{abstract}
Knowledge of the variant anatomy of the intradural venous sinuses is important to anatomists and clinicians alike. Herein, we report a cadaveric case of the rare venous sinus of Kelch, which some have believed is a remnant of the cranioorbital sinuses. To our knowledge, only one other cadaveric case has been reported in the extant medical literature. Clinically, knowledge of such a variant venous sinus can minimize misdiagnoses such as when anatomical variations are noted on imaging. Surgically, such an understanding can avoid intraoperative complications such as iatrogenic hemorrhage.
\end{abstract}

Key words: Anatomical variation, Dural venous sinus, Venous sinus of Kelch, Cadaver, Anatomy

Received February 25, 2021; Accepted April 5, 2021

\section{Introduction}

Unique anatomy and differences in venous drainage of the cranium are important considerations for the clinician and observation of these aberrations may be important in diagnosis and treatment. Clinical cases involving the spread of infection from the middle ear to the intracranial venous system, as well as cases involving chronic otitis media, have all presented with an abnormal presence of a petrosquamous sinuses [1,2]. Here the authors report an unusual finding of a bilateral presentation of a rare venous sinus, the venous sinus of Kelch.

\section{Case Report}

During the routine dissection of a male cadaver aged 75 -year-old at death, a venous sinus (Fig. 1) was identified

\section{Corresponding author:}

Joe Iwanaga (i)

Department of Neurosurgery, Tulane Center for Clinical Neurosciences, Tulane University School of Medicine, New Orleans, LA 70112, USA

E-mail: iwanagajoeca@gmail.com traveling from the superior orbital fissure anteriorly, over the floor of the middle cranial fossa, over the petrous part of the temporal bone, to drain into the transverse sinus. The

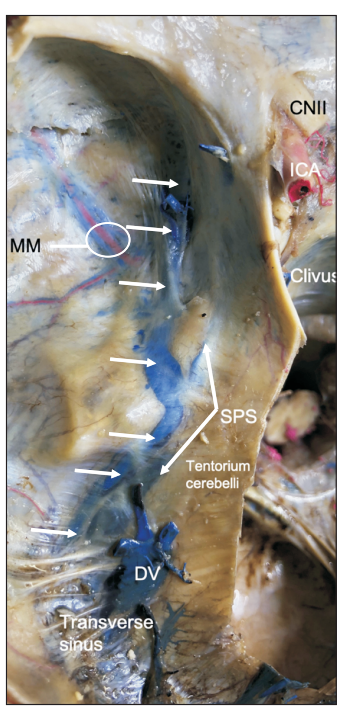

Fig. 1. Cadaveric dissection of a left-sided venous sinus of Kelch (arrows). Note the middle meningeal veins and artery (MM), superior petrosal sinus (SPS), draining vein (DV), optic nerve (CNII), and internal carotid artery (ICA) for reference.

\section{Copyright (c) 2021. Anatomy \& Cell Biology}

This is an Open Access article distributed under the terms of the Creative Commons Attribution Non-Commercial License (http://creativecommons.org/licenses/by-nc/4.0/) which permits unrestricted non-commercial use, distribution, and reproduction in any medium, provided the original work is properly cited. 
variant was found on left and right sides (Fig. 2) but the right sided sinus was damaged before it could be photographed. Anteriorly, the sinus communicated with the superior ophthalmic vein, which drained primarily into the cavernous sinus. Along the floor of the middle cranial fossa, the venous sinuses communicated with the middle meningeal veins (Fig. 1). The superior petrosal sinus traveled medial to the sinus and did not communicate with it. The variant venous sinus was approximately $8 \mathrm{~mm}$ in width at its anterior and posterior extremes but was dilated to $1.2 \mathrm{~cm}$ as it crossed over the petrous part of the temporal bone. The sinuses were roughly $7.2 \mathrm{~cm}$ long. No additional anatomical variations or intracranial pathology were noted in this specimen.

\section{Discussion}

The dural venous sinus of Kelch is a rare venous sinus of the skull base and one which has been infrequently reported. It effectively unites the veins of the superior orbital fissure to the transverse sinus. To our knowledge, cadaveric findings of this sinus have only been reported once before and these were of a variant of the sinus. Moreover, a bilaterally present sinus has not been reported.

\section{Embryology}

In the early embryo at the 5 to $8 \mathrm{~mm}$ stage, the brain is covered by a continuous plexus which drains both laterally and ventrally through three stems, the anterior, the middle, and the posterior. By the 11 to $14 \mathrm{~mm}$ embryonic stage, each subdivision of the brain is drained laterally into the dural plexuses by primitive pia-arachnoidal vessels. During the

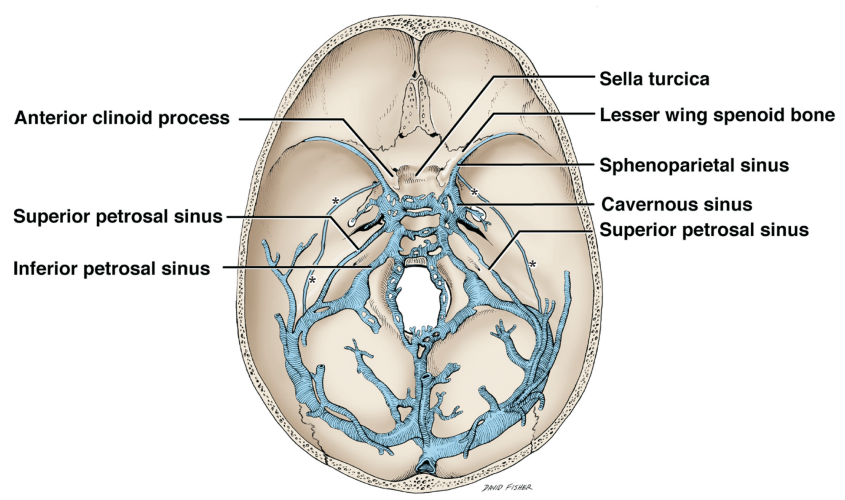

Fig. 2. Schematic drawing of the bilateral venous sinuses of Kelch (asterisk) joining veins of the superior orbital fissure to the transverse sinus.
17 to $20 \mathrm{~mm}$ stage of development, middle and posterior plexuses have joined to form the sigmoid sinus while the two parts of the anterior dural plexus; the tentorial sinus, and the marginal sinus border the expanding hemisphere caudally. At the 22 to $24 \mathrm{~mm}$ embryonic stage, the supraorbital vein no longer drains into the stem of the anterior dural sinus, which has diminished and is continuous with the prootic sinus. The prootic sinus is a stem of the middle dural plexus which incorporates into the stem of the posterior dural plexus and empties into the sigmoid sinus. Growth of the brain has elongated the tentorial sinus, and the marginal sinus now constitutes the medial end of the primitive transverse sinus.

By the $40 \mathrm{~mm}$ stage, the cavernous sinuses are developing, and are medial extensions of the prootic sinus which is now continuous with the newly developed inferior petrosal sinus. A new lateral tributary of the prootic sinus now anastomoses with a primitive temporal emissary vein derived from the sigmoid sinus to form the petrosquamous sinus. At the 60 to $80 \mathrm{~mm}$ stage, cerebral growth and the development of the otic capsule has led to the formation of the superior petrosal sinus. The primitive transverse sinus has moved backward on the sigmoid sinus and has anastomosed with the tentorial sinus. The prootic sinus is now continuous with the petrosquamous sinus. In the adult, remnants of the tentorial sinus may persist and anastomose with the cavernous sinus which is now joined with the superior petrosal sinus. It would be at this stage that one would expect to find the sinus of Kelch being formed (Fig. 3). The petrosquamous sinus and the remnants of the prootic sinus become diploic veins and drain

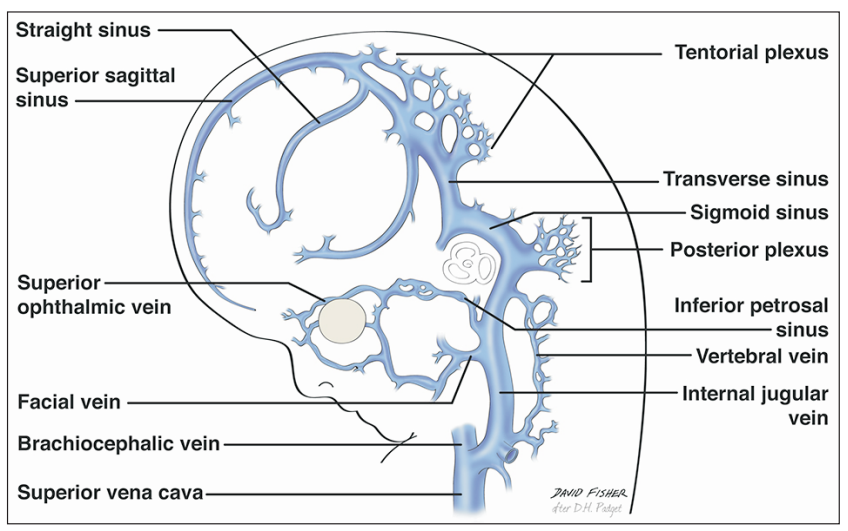

Fig. 3. Fetal development of the intracranial venous sinuses. In this schematic drawing, note that the superior ophthalmic vein are developing and are near the more posteriorly located transverse sinus. This position would be the most likely stage where communication (dotted line) could occur between these two systems e.g., the venous sinus of Kelch. 
the dura and bone when the middle meningeal sinus drain through the foramen ovale and into the sagittal sinus [3].

\section{Comparative anatomy}

In primates, there are large evolutionary differences in the orbitotemporal venous sinuses. In primitive primates, these sinuses are large and drain the brain. The cranio-orbital sinus empties into the postglenoid emissary vein which also receives drainage from the petrosquamous sinus. In humans, this network of orbitotemporal sinuses is modified and persists as part of the middle meningeal vein system which no longer drains the brain, the orbit, or the temporal fossa, and the petrosquamous sinus is attenuated or non-existent [4]. Due to these evolutionary differences, the presence of a venous sinus of Kelch may indicate the remnant of the cranioorbital sinus in humans [5].

\section{Sinus of Kelch}

Reports of a venous sinus of Kelch are rare in the literature. Knott [6] briefly described such a sinus as an accessory sinus which passes backward from the superior orbital fissure over the upper border of the petrous part of the temporal bone to terminate into the transverse sinus. Hollinshead [7] stated similarities between the sinus of Hyrtl and the sinus of Kelch and indicated that they may actually describe the same structure. More recently, unilaterally present sinus of Hyrtl have been described in the literature and bilateral occurrences appear to have not been previously reported [8-10]. We previously reported a unilateral variant of the sinus of Kelch found in an 88-year-old at death female cadaver [11]. In this specimen, the sinus of Kelch, which was approximately $3 \mathrm{~mm}$ in diameter posteriorly and $6 \mathrm{~mm}$ in diameter anteriorly, drained posteriorly into the superior petrosal sinus rather than into the transverse sinus. On the ipsilateral side of this same specimen, an enlarged arachnoid granulation was identified protruding through the foramen rotundum. However, in the case described herein, the only intracranial anatomical variations identified were the two venous sinuses of Kelch.

In conclusion, knowledge of the variant anatomy of the intradural venous sinuses is important to anatomists and clinicians alike. Clinically, such knowledge can minimize misdiagnoses such as when anatomical variations are noted on imaging. Surgically, such an understanding can avoid intraoperative complications such as iatrogenic hemorrhage.

\section{ORCID}

Mitchell Couldwell:

https://orcid.org/0000-0002-9981-0788

Aimee Aysenne: https:/orcid.org/0000-0003-4183-1161

Joe Iwanaga: https://orcid.org/0000-0002-8502-7952

Aaron S. Dumont: https://orcid.org/0000-0002-8077-8992

R. Shane Tubbs: https://orcid.org/0000-0003-1317-1047

\section{Author Contributions}

Conceptualization: RST, JI. Data acquisition: RST, JI. Data analysis or interpretation: MC, AA, RST. Drafting of the manuscript: MC, AA, JI. Critical revision of the manuscript: ASD, RST. Approval of the final version of the manuscript: all authors.

\section{Conflicts of Interest}

No potential conflict of interest relevant to this article was reported.

\section{Acknowledgements}

The authors sincerely thank those who donated their bodies to science so that anatomical research could be performed. Results from such research can potentially increase mankind's overall knowledge that can improve patient care. Therefore, these donors and their families deserve our highest gratitude [12].

\section{References}

1. Klein NE. Catalog of human variations. Plast Reconstr Surg 1985;76:478.

2. An YH, Wee JH, Han KH, Kim YH. Two cases of petrosquamosal sinus in the temporal bone presented as perioperative finding. Laryngoscope 2011;121:381-4.

3. Padget DH. The cranial venous system in man in reference to development, adult configuration, and relation to the arteries. Am J Anat 1956;98:307-55.

4. Diamond MK. Homology and evolution of the orbitotemporal venous sinuses of humans. Am J Phys Anthropol 1992;88:21144.

5. Streeter GL. The development of the venous sinuses of the dura mater in the human embryo. Am J Anat 1915;18:145-78.

6 . Knott JF. On the cerebral sinuses and their variations. J Anat Physiol 1881;16(Pt 1):27-42. 
7. Hollinshead WH. Anatomy for surgeons. 3rd ed. Philadelphia: Lippincott; 1982.

8. Tubbs RS. Anatomy, imaging and surgery of the intracranial dural venous sinuses. Philadelphia: Elsevier; 2019.

9. Tubbs RS, Loukas M, Shoja MM, Oakes WJ. Accessory venous sinus of Hyrtl. Folia Morphol (Warsz) 2007;66:198-9.

10. Cvetko E, Bosnjak R. Unilateral absence of foramen spinosum with bilateral ophthalmic origin of the middle meningeal artery: case report and review of the literature. Folia Morphol (Warsz) 2014;73:87-91.
11. Umeh R, Loukas M, Oskouian RJ, Tubbs RS. Ectopic arachnoid granulation involving a rare intracranial venous sinus variant. Folia Morphol (Warsz) 2017;76:319-21.

12. Iwanaga J, Singh V, Ohtsuka A, Hwang Y, Kim HJ, Moryś J, Ravi KS, Ribatti D, Trainor PA, Sañudo JR, Apaydin N, Şengül G, Albertine KH, Walocha JA, Loukas M, Duparc F, Paulsen F, Del Sol M, Adds P, Hegazy A, Tubbs RS. Acknowledging the use of human cadaveric tissues in research papers: recommendations from anatomical journal editors. Clin Anat 2021;34:24. 